\title{
Infección natural por Strongyloides stercoralis en Pithecia monachus (Primates, Pitheciidae). Primer reporte en el Perú
}

\author{
Natural infection by Strongyloides stercoralis in Pithecia monachus \\ (Primates, Pitheciidae). First report in Peru
}

\author{
Manuel Tantaleán V..$^{1,4}$, Nofre Sánchez P. ${ }^{2}$, Carlos Marcial Perea ${ }^{3}$
}

\section{Resumen}

El objetivo del presente trabajo fue identificar unos nematodos obtenidos post mortem del intestino delgado de Pithecia monachus criado en cautiverio en Iquitos, Perú. La identificación se hizo observando la morfología y anatomía de los especímenes hembras del intestino del hospedero y de machos y hembras obtenidos por cultivo. Todos los especímenes se identificaron como de Strongyloides stercoralis. Se concluye que esta especie se encuentra por primera vez en un primate en el Perú.

Palabras clave: Phitecia monachus; Strongyloides stercoralis; Perú

\section{Abstract}

The objective of the present work was to identify nematodes obtained post mortem from the small intestine of Pithecia monachus bred in captivity in Iquitos, Peru. The identification was made observing the morphology and anatomy of the female specimens of the intestine of the host and of males and females obtained by culture. All specimens were identified as Strongyloides stercoralis. It is concluded that this species is found for the first time in a primate in Peru.

Key words: Phitecia monachus; Strongyloides stercoralis; Peru

\footnotetext{
${ }^{1}$ Laboratorio de Parasitología en Fauna Silvestre y Zoonosis, Facultad de Ciencias Biológicas, Universidad Nacional Mayor de San Marcos, Lima, Perú

${ }^{2}$ Instituto Veterinario de Investigaciones Tropicales y de Altura (IVITA), Universidad Nacional Mayor de San Marcos, Lima, Perú

${ }^{3}$ Instituto de Investigaciones de la Amazonía Peruana, Iquitos, Perú

${ }^{4}$ E-mail: mtantaleanv1@gmail.com
} 


\section{INTRODUCCIÓN}

Strongyloides stercoralis es un nematode que pertenece al Orden Rhabditida y familia Strongyloididae (Anderson, 2000). Es una especie cosmopolita, parásita de humanos, trasmitida por el suelo en áreas tropicales y subtropicales, hiperendémica en varias zonas de la selva peruana y con menor grado en la costa y sierra (Egido et al., 2001; Marcos et al., 2010). En un estudio de hepatitis virales, protozoos y helmintos en seis especies de primates en Brasil, se encontró que el $17.2 \%$ de las muestras fecales estudiadas tenían S. stercoralis (Pereira et al., 2010). Este nematodo también se encuentra en primates cercopitecoideos (Cercopithecidae) en Costa de Marfil (Kouassi et al., 2015).

Pithecia monachus es un primate sudamericano, frugívoro, depredador de semillas, nueces y en menor proporción de insectos. En el Perú, se distribuye en el nororiente, Loreto y Ucayali (Marsh, 2014); prefiere los árboles de gran altura y puede vivir hasta 25 años en cautiverio y llegar a medir $50 \mathrm{~cm}$. Son de hábitos diurnos. La investigación parasitológica en este primate es casi nula en el Perú.

El objetivo del presente estudio fue dar a conocer el primer caso en el país de infección natural con Strongyloides stercoralis, un nematodo parásito de humanos, en Phitecia monachus.

\section{Materiales y MéTodos}

Una hembra juvenil de aproximadamente nueve meses de edad, de la especie Pithecia monachus (huapo negro), fue comprada en el pueblo de Mazán ( $3^{\circ} 29244.843$ $\mathrm{S}, 73^{\circ} 5243.653 \mathrm{~W}$ ), provincia de Maynas, región Loreto, a 30 minutos de la ciudad de Iquitos, donde vivió con una familia de personas durante dos meses. Ingresó al Centro de Rescate Amazónico (CREA) en Iquitos en agosto de 2015, permaneciendo en cautive- rio durante siete meses, donde murió. Durante su permanencia en el Centro de Rescate compartió una jaula con tres perezosos adultos y dos primates (Saimiri sciureus, fraile y Cebus apella, machín blanco) y se le alimentó con un balanceado y frutas de la región tres veces al día.

En la necropsia se encontró un animal pálido y muy delgado (caquéctico). El tracto gastrointestinal contenía abundante mucosidad con restos alimenticios y gran cantidad de nematodos hembras, muy pequeñas, y abundantes huevos. Los demás órganos estaban pálidos y sin otras alteraciones aparentes.

Una porción del contenido intestinal se colocó en una placa de Petri con agua corriente esterilizada para observar el desarrollo de los huevos y la formación de adultos, macho y hembra, de la generación de vida libre. Las preparaciones microscópicas de 12 (7 machos y 5 hembras) parásitos de vida libre se fijaron previamente con formol al 10\% y para su estudio se clarificaron con lactofenol de Amann durante 24 horas (LamotheArgumedo, 1997).

La identificación de la especie se hizo comparando la morfología y las estructuras anatómicas de los especímenes colectados con los criterios taxonómicos de identificación previamente publicados (Little, 1966; Grove, 1996). Las medidas se expresan en milímetros, con el rango entre paréntesis. Las fotografías se tomaron en un microscopio Leica DM 750 con cámara digital incorporada. El material se encuentra archivado en la colección del Laboratorio de Parasitología en Fauna Silvestre y Zoonosis, Facultad de Ciencias Biológicas, Universidad Nacional Mayor de San Marcos, Lima.

\section{Resultados}

En la placa con el cultivo de los huevos se desarrollaron estadios adultos, tanto de machos como hembras de la generación de vida libre. En la identificación de los 


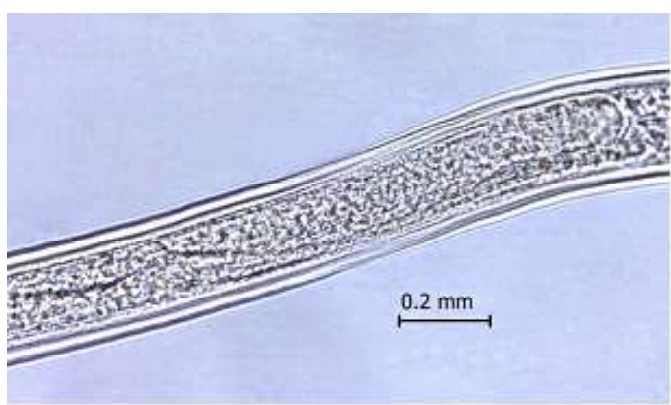

(A)

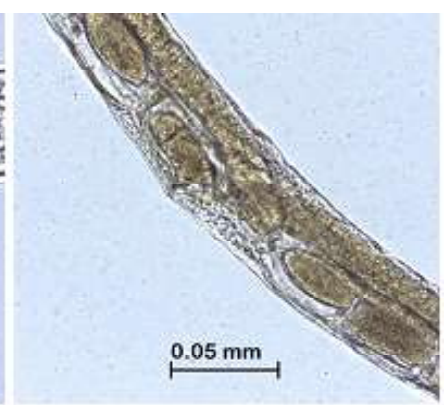

(B)

Figura 1. Strongyloides stercoralis en Pithecia monachus. A: Hembra parásita. Inıcio y recorrido del ovario; B: Hembra de vida libre. Región de la vulva y huevos en útero

especímenes obtenidos se observó que los ovarios en la hembra parásita eran paralelos al intestino y no en espiral alrededor de él, como sucede en S. cebus (Figura 1A), única especie presente en primates sudamericanos. Así mismo, se observó que los labios vulvares en la hembra de vida libre no eran salientes (Figura 1B), además de la disposición de las papilas caudales y detalle de la membrana espicular en el macho, por lo que se identificaron como $S$. stercoralis.

\section{Breve Descripción de los Especímenes}

\section{Hembra parásita}

Mide $2.3 \mathrm{~mm}$ (2.1-2.8) de largo por $0.036 \mathrm{~mm}(0.028-0.039)$ de ancho. Delgadas, transparentes, con cola corta. El esófago filariforme mide $0.145 \mathrm{~mm}(0.128-0.146)$ y está dividido en dos secciones, la muscular que mide aproximadamente la cuarta parte y la glandular. Las dos ramas ováricas y uterinas son rectas y corren paralelas al intestino, contienen pocos huevos, entre 3 y 5 . La vulva se abre en el tercio posterior del cuerpo. Los huevos, sin segmentación en útero, son muy parecidos a los de la generación de vida libre y miden $0.06 \mathrm{~mm}(0.04-0.07)$.

\section{Macho de la generación de vida libre}

Mide $0.93 \mathrm{~mm}(0.81-1.1)$ de longitud por $0.047 \mathrm{~mm}$ (0.039- 0.052), con el extremo posterior curvado hacia la cara ventral. Esófago rhabditoide. Espículas iguales puntiagudas y con poca curvatura en la que lleva una pequeña membrana recta, cada espícula tiene dos engrosamientos laterales. El gubernáculo con dos extensiones laterales; las papilas caudales son muy pequeñas, de las cuales las pre-anales sub-ventrales se disponen por detrás de la papila única pre-anal y las pos-anales sub-ventrales están cerca de las pos-anales sub-dorsales.

\section{Hembra de la generación de vida libre}

Mide $1.3 \mathrm{~mm}(0.92-1.8)$ de longitud por $0.056 \mathrm{~mm}(0.053-0.081)$. Esófago rhabditoide. Leve contracción por detrás de la vulva cuyos labios no sobresalen. Aparato genital didelfo, los ovarios se doblan sobre ellos mismos, útero con dos ramas, anterior y posterior y con varios huevos.

\section{Discusión}

S. stercoralis es un nematodo parásito de humanos en las zonas tropicales y subtropicales del mundo y es trasmitido por el suelo en lugares con escaso saneamiento ambiental. Se calcula que afecta entre $30 \mathrm{y}$ 100 millones de personas (Kakati et al., 2011). En los primates del nuevo mundo, la especie parásita es $S$. cebus y en los del viejo mundo es $S$. füelleborni, una subespecie de esta última. S. füelleborni kellyi parasita 
a humanos en África y Papua Nueva Guinea (Viney et al., 1991).

Se ha explicado que la infección de los primates sudamericanos con parásitos provenientes de humanos se ve favorecida por la tendencia del hombre a mantenerlos en cautiverio, y a que estos animales pueden vivir cerca de las poblaciones humanas (Michaud et al., 2003; Botero et al., 2011).

La infección natural por $S$. stercoralis se observa en primates no humanos Cercopithecidae en Costa de Marfil (África) como Cercocebus atys, Cercopithecus campbelli, C. diana, C. petaurista y Procolobus verus, con prevalencias que oscilan entre 2.3 y $17.8 \%$ (Kouassi et al., 2015). Del mismo modo, Erithrocebus pata del Viejo Mundo es susceptible a $S$. stercoralis (Barrett et al., 1988). Penner (1981) reportó tres casos de autoinfección fatal con S. stercoralis en Gorilla gorilla y Pan troglodytes ocurridos en el Zoológico de San Diego (EEUU). En Sudamérica no hay investigaciones confirmadas de infección natural de primates con $S$. stercoralis, porque los reportes solo se refieren a resultados de estudios coprológicos; sin embargo, al estudiar el comportamiento de Callithrix penicillata como animal modelo para investigar aspectos clínicos y patológicos de la infección por $S$. stercoralis, se concluyó que este primate es susceptible a la infección (Mati et al., 2014).

En el presente caso, y considerando el modo de vida de $P$. monachus, el animal se habría infectado cuando estaba en posesión humana, teniendo en consideración que la larva infectante $\mathrm{L}_{3}$ se desarrolla en el suelo y penetra la piel del hospedero. La hiperinfección en el animal se produjo, probablemente, aprovechando una posible condición de limitada resistencia. La fauna parasitaria de $P$. monachus no es muy conocida en el Perú y solo se menciona un caso de infección por Ascaris lumbricoides en Iquitos (Sarmiento et al., 1999).
En conclusión, por observación y estudio de las características morfo-anatómicas, se identifica por primera vez a $S$. stercoralis en $P$. monachus procedente de Iquitos, Perú.

\section{Literatura Citada}

1. Anderson RC. 2000. Nematode parasites of vertebrates: their development and transmission. $2^{\text {nd }}$ ed. Oxford, UK: CABI Publishing. 650 p.

2. Barrett KE, Neva FA, Gam AA, Cicmanec J, London WT, Phillips JM, Metcalfe DD. 1988. The immune response to nematode parasites: modulation of mast cell numbers and function during Strongyloides stercoralis infections in nonhuman primates. Am J Trop Med Hyg 38: 574-581. doi: 10.4269/ajtmh.1988.38.574

3. Botero LC, Fernández A, Forero N, Rosas GS, Soler-Tobar D. 2011. Análisis retrospectivo de las enfermedades parasitarias del mono ardilla (Saimiri sciureus) en dos condiciones ex situ en el noroccidente de los Andes sudamericanos. Rev Med Vet 22: 85-93. doi: 10.19052/mv.557

4. Egido JM, De Diego JA, Penin P. 2001. The prevalence of enteropathy due to strongyloidiasis in Puerto Maldonado (Peruvian Amazon). Braz J Infect Dis 5: 119-123. doi: 10.1590/ S1413-86702001000300003

5. Grove DI. 1996. Human strongyloidiasis. Adv Parasit 38: 251-309. doi: 10.1016/S0065-308X(08)60036-6

6. Kakati B, Dang S, Heif M, Kadarine K, McNight W, Aduli F. 2011. Strongyloides duodenitis: case report and review of literature. J Natl Med Assoc 103: 6063. doi: 10.1016/S0027-9684(15)-30246-7

7. Kouassi RY, McGraw SW, Yao PK, Abou-Bacar A, Brunet J, Pesson B, Bonfoh B, et al. 2015. Diversity and prevalence of gastrointestinal parasites in seven non-human primates of the Taï National Park, Côte d'Ivoire. Parasite 22(1). doi: 10.1051/parasite/2015001 
8. Lamothe-Argumedo R. 1997. Manual de técnicas para preparar y estudiar los parásitos de animales silvestres. México DF: AGT. 43 p.

9. Little MD. 1966. Comparative morphology of six species of Strongyloides (Nematoda) and redefinition of the genus. J Parasitol 52: 69-84 doi: 10.2307/ 3276396

10. Marcos LA, Cabrera R, Machicado JD, Canales M, Terashima A. 2010. Distribution of prevalence of Strongyloides stercoralis in Peru (1981-2010): an exploratory study. Rev Peru Parasitol 18: 39-49.

11. Marsh LK. 2014. A Taxonomic Revision of the Saki monkeys, Pithecia Desmarest, 1804. Neotrop Primates 21: 1-162. doi: 10.1896/044.021.0101

12. Mati VL, Raso P, de Melo AL. 2014. Strogyloides stercoralis infection in marmosets: replication of complicated and un complicated human disease and parasite biology. Parasite Vector 7: 579. doi: 10.1186/s13071-014-0579-2

13. Michaud C, Tantaleán M, Ique C, Montoya E, Gozalo A. 2003. A survey for helminth parasites in feral New World non-human primate populations and its comparison with parasitological data from man in the region. J Med Primatol 32: 341-345. doi: 10.1046/j.16000684.2003.00037.x

14. Pereira WL, Rocha G, da Silva KS, Pereira MC, Moreira M. 2010. Ocurrencia de hepatitis virales, helmintiasis y protozoosis en primates neotropicales procedentes de cría domiciliaria: afecciones de transmisión fecal-oral con potencial zoonótico. Rev Pan-Amazon Saude 1: 57-60. doi: 10.5123/S217662232010000300008

15. Penner LR. 1981. Concerning threadworm (Strongyloides stercoralis) in great apes-lowland gorillas (Gorilla gorilla) and chimpanzees (Pan troglodytes). J Zoo Anim Med 12: 128-131. doi: $10.2307 / 20094543$

16. Sarmiento L, Tantaleán M, Huiza A. 1999. Nemátodos parásitos del hombre y de los animales en el Perú. Rev Per Parasitol 14: 9-65.

17. Viney ME, Ashford RW, Barnish G 1991. A taxonomic study of Strongyloides Grassi, 1879 (Nematoda) with special reference to Strongyloides fuelleborni Von Linstow, 1905 in man in Papua New Guinea and the description of a new subspecies. Syst Parasitol 18: 95-109. doi: 10.1007/BF00017661 